\title{
Video Article \\ Isolation and Characterization of Mesenchymal Stromal Cells from Human Umbilical Cord and Fetal Placenta
}

\author{
Naimisha Beeravolu ${ }^{1,2}$, Christina McKee ${ }^{1,2}$, Ali Alamri ${ }^{1,2}$, Sasha Mikhael ${ }^{3}$, Christina Brown ${ }^{1,2}$, Mick Perez-Cruet ${ }^{2,4}$, G. Rasul Chaudhry ${ }^{1,2}$ \\ ${ }^{1}$ Department of Biological Sciences, Oakland University \\ ${ }^{2}$ OU-WB Institute for Stem Cell and Regenerative Medicine \\ ${ }^{3}$ Department of Obstetrics and Gynecology, St. John Provindence - Providence Park Hospital \\ ${ }^{4}$ Department of Neurosurgery, Beaumont Health System
}

Correspondence to: G. Rasul Chaudhry at chaudhry@oakland.edu

URL: https://www.jove.com/video/55224

DOI: doi: $10.3791 / 55224$

Keywords: Developmental Biology, Issue 122, Perinatal, Umbilical cord, Cord-placenta junction, Placenta, Mesenchymal stromal cells, MSC markers, Multipotent cells, Pluripotent markers

Date Published: 4/3/2017

Citation: Beeravolu, N., McKee, C., Alamri, A., Mikhael, S., Brown, C., Perez-Cruet, M., Chaudhry, G.R. Isolation and Characterization of Mesenchymal Stromal Cells from Human Umbilical Cord and Fetal Placenta. J. Vis. Exp. (122), e55224, doi:10.3791/55224 (2017).

\section{Abstract}

The human umbilical cord (UC) and placenta are non-invasive, primitive and abundant sources of mesenchymal stromal cells (MSCs) that have increasingly gained attention because they do not pose any ethical or moral concerns. Current methods to isolate MSCs from UC yield low amounts of cells with variable proliferation potentials. Since UC is an anatomically-complex organ, differences in MSC properties may be due to the differences in the anatomical regions of their isolation. In this study, we first dissected the cord/placenta samples into three discrete anatomical regions: UC, cord-placenta junction (CPJ), and fetal placenta (FP). Second, two distinct zones, cord lining (CL) and Wharton's jelly (WJ), were separated. The explant culture technique was then used to isolate cells from the four sources. The time required for the primary culture of cells from the explants varied depending on the source of the tissue. Outgrowth of the cells occurred within 3 - 4 days of the CPJ explants, whereas growth was observed after 7 - 10 days and 11 - 14 days from CL/WJ and FP explants, respectively. The isolated cells were adherent to plastic and displayed fibroblastoid morphology and surface markers, such as CD29, CD44, CD73, CD90, and CD105, similarly to bone marrow (BM)-derived MSCs. However, the colony-forming efficiency of the cells varied, with CPJ-MSCs and WJ-MSCs showing higher efficiency than BM-MSCs. MSCs from all four sources differentiated into adipogenic, chondrogenic, and osteogenic lineages, indicating that they were multipotent. CPJ-MSCs differentiated more efficiently in comparison to other MSC sources. These results suggest that the CPJ is the most potent anatomical region and yields a higher number of cells, with greater proliferation and self-renewal capacities in vitro. In conclusion, the comparative analysis of the MSCs from the four sources indicated that CPJ is a more promising source of MSCs for cell therapy, regenerative medicine, and tissue engineering.

\section{Video Link}

The video component of this article can be found at https://www.jove.com/video/55224/

\section{Introduction}

Stem cells are present in various organs and tissues of the body. They possess regenerative potential and play a major role in repairing damaged tissues in the body throughout the span of the human life ${ }^{1,2}$. This has generated a great deal of interest in adult stem cells (ASCs), particularly since, unlike embryonic stem cells (ESCs), the use of ASCs does not pose moral and ethical dilemmas. Several studies have reported the isolation of ASCs, such as mesenchymal stromal cells (MSCs); hematopoietic stem cells (HSCs); and different progenitors, including various adult sources ranging from bone marrow (BM) to adipose tissue (AT) and dental pulp ${ }^{3,4,5}$. However, the number of ASCs present in most of the adult niches is limited, and their isolation generally involves an invasive and painful procedure with possible donor site morbidity. In addition, donor age and environmental stress could also play a significant role in determining the quality and biological activity of the isolated cells ${ }^{6,7,8}$. ASCs also display limited proliferation and differentiation potential during culture in vitro.

To overcome these disadvantages of current ASCs, new sources have been pursued to isolate stem cells. These efforts have led to the isolation of stem cells from perinatal sources, including cord blood, cord tissue, placenta, and amniotic fluid ${ }^{9}$. These sources have gained attention because of their easy and abundant availability ${ }^{10,11,12,13}$. Furthermore, perinatal tissues can be obtained non-invasively, and stem cells derived from them are more primitive than ASCs isolated from adult sources ${ }^{14,15}$. They are isolated from the tissues obtained at birth and are considered to have undergone minimal alterations in the genome due to aging and environmental stresses ${ }^{16}$.

However, the reported characteristics of stem cells from perinatal sources and their potential to self-renew as well as to differentiate vary widely ${ }^{11,17}$. This could be in part due to the fact that the human umbilical cord (UC) is a complex organ. We hypothesize that the discrete regions of perinatal tissue create specific niches responsible for the variations and more primitive nature of these stem cells in comparison to ASCs derived from non-perinatal sources. This study describes the dissection of cord/placenta samples into three discrete anatomical regions: UC, 
cord-placenta junction (CPJ), and fetal placenta (FP). The UC was further dissected into two zones: cord lining (CL) and Wharton's Jelly (WJ). Analysis of the isolated cells from CL, WJ, CPJ, and FP demonstrated that they all exhibited fibroblastoid morphology and expressed MSC markers, but they differed in their self-renewal and differentiation potential. CPJ-derived cells exhibited a higher rate of proliferation and selfrenewal potential as compared to UC- and FP-derived cells, making them a more promising source for cell therapy, regenerative medicine, and tissue engineering.

\section{Protocol}

Samples $(n=3)$ were obtained from the consenting, healthy donors through the Beaumont Hospital BioBank, Royal Oak, MI and the Providence Hospital, Southfield, MI under HIC- (HIC \#2012-101) and IRB- (IRB\# 820662-3), respectively, approved protocols and processed as approved by the IBC (\#2858) of Oakland University, Rochester, MI.

\section{Processing Human Umbilical Cord, Cord-placenta Junction, and Fetal Placenta and Isolating Cells}

1. Aseptically collect samples of UC approximately $10 \mathrm{~cm}$ in length, including $5-6 \mathrm{~cm}$ connected to the CPJ and $3-4 \mathrm{~cm}$ of FP. Immediately place each sample in a collection cup containing medium (DMEM with $4,500 \mathrm{mg} / \mathrm{mL}$ glucose and antibiotic solution $(0.1 \%$ gentamicin, $0.2 \%$ streptomycin, and $0.12 \%$ penicillin)) and store at $4{ }^{\circ} \mathrm{C}$ until it is transported to the lab by placing it on ice in a Styrofoam box. Process the sample at $4{ }^{\circ} \mathrm{C}$ within $2-4 \mathrm{~h}$ of collection.

2. Place the sample in a 150-mm Petri dish kept on ice in a biosafety cabinet. Using a needle and syringe, rinse it multiple times with ice-cold PBS to remove blood clots. Make sure the sample is kept wet with PBS during processing and do not let it dry. To maintain sterility, handle the sample aseptically using autoclaved PBS and surgical tools throughout sample processing.

3. Carefully examine the sample to identify different anatomical regions: UC, CPJ, and FP. First dissect the UC. Hold the fetal end of the UC with forceps and carefully make the first cut just at the top of the CPJ using a pair of scissors. Make the second cut below the CPJ to separate the CPJ $(1.5-2.0 \mathrm{~cm})$ from the FP. Place the separated UC, CPJ, and FP in separate Petri dishes for further processing.

4. Cut the UC longitudinally with the help of scissors and forceps, completely exposing the blood vessels and surrounding WJ without disturbing the epithelium.

5. Scrape the WJ away from the blood vessels and inner epithelium of the subamnion using a scalpel, and then remove the blood vessels. Be sure to collect any remaining perivascular WJ under and around the blood vessels, and place the collected WJ in a separate Petri dish.

6. Collect the remaining tissue, cord lining $(\mathrm{CL})$, in a separate Petri dish.

7. After separation of the tissues representing CL, WJ, CPJ, and FP, replace the PBS with $3-5 \mathrm{~mL}$ of commercial trypsin solution and separately cut each of the tissues into 1 - to $2-\mathrm{mm}$ pieces using scissors.

8. Incubate the tissue pieces in commercial trypsin solution at $37^{\circ} \mathrm{C}$ for $30 \mathrm{~min}$ in a $5 \% \mathrm{CO}_{2}$ incubator for partial digestion of the samples Observe the partial digestion by visualizing release of cells using a phase contrast microscope.

9. To the partially-digested samples, add an equal volume of culture medium (CM; DMEM with 4,500 mg/mL glucose and $2 \mathrm{mM} \mathrm{L-glutamine,}$ supplemented with $10 \%$ human serum/FBS and antibiotic solution $(0.1 \%$ gentamicin, $0.2 \%$ streptomycin, and $0.12 \%$ penicillin) $)$ to neutralize the commercial trypsin solution. Transfer the content into $50-\mathrm{mL}$ centrifuge tubes and let the partially-digested tissue pieces settle for 3 min.

10. Carefully aspirate the supernatant, including single cells (they do not expand efficiently), and plate $15-20$ partially-digested tissue pieces on a $75-\mathrm{cm}^{2}$ tissue culture flask and add $9 \mathrm{~mL}$ of $\mathrm{CM}$. Incubate at $37^{\circ} \mathrm{C}$ and do not disturb the culture flasks for $2-3$ days to allow for the adherence of tissue pieces. NOTE: The remaining portion of the partially-digested tissue samples can be cryopreserved for the future isolation of cells.

11. After 3 days of incubation, change the $\mathrm{CM}$ and look for the appearance of outgrowth of cells from the explant on a daily basis; cell growth should be evident from the adherent explants after 3 - 4 days, 7 - 10 days, and 11 - 14 days of incubation for CPJ, CL/WJ, and FP, respectively. From this point onwards, change the CM after every 3 days or as needed. NOTE: Cell growth reaches $70 \%$ confluence 7 - 10 days after the initial cell outgrowth from the explant. Note that non-adherent tissue pieces will not give rise to any cell outgrowth until they adhere to the plastic. Care must be taken so that the flask is not disturbed in order to avoid the detachment of the adherent tissue pieces. If there are any floating pieces after the first 3 days of culturing, they could be rescued by transferring them to a new flask for attachment.

NOTE: When cell growth from the tissue explants reaches $70 \%$ confluence, they should be subcultured. As stated above, MSCs from CL/WJ, FP, CPJ and will reach confluence in $10-14$ days, $14-20$ days, and $17-24$ days, respectively.

12. To subculture outgrown cells from the explants, dissociate the cells by using $1-2 \mathrm{~mL}$ of commercial trypsin solution/75-cm ${ }^{2}$ culture flask and incubate at $37^{\circ} \mathrm{C}$ for $3 \mathrm{~min}$. Neutralize with $1-2 \mathrm{~mL}$ of $\mathrm{CM}$ and centrifuge $1,500 \times \mathrm{g}$ for $3 \mathrm{~min}$. Make sure to rotate the flask while adding trypsin for even spreading throughout.

13. NOTE: The pelleted cells are considered passage $0(P 0)$ and will be suspended in $\mathrm{CM}$, counted, and subcultured at a seeding density of $1 \mathrm{x}$ $10^{4} / \mathrm{cm}^{2}$ for amplification, characterization, and cryopreservation. The excess cells can be cryopreserved at P0.

\section{Characterizing the Isolated Cells}

\section{Colony forming efficiency (CFE) assay}

1. Coat the $100-\mathrm{mm}$ Petri dishes (surface area of $60 \mathrm{~cm}^{2}$ ) with $0.1 \%$ gelatin for $30 \mathrm{~min}$ and place them in the $\mathrm{CO}_{2}$ incubator at $37^{\circ} \mathrm{C}$ NOTE: Although not necessary, gelatin-coating improves cell adherence.

2. Remove the excess gelatin and seed the coated plate with P0 cells at a concentration of $1.63 \mathrm{cells} / \mathrm{cm}^{2}$. Add $3 \mathrm{~mL}$ of $\mathrm{CM}$ and incubate in the $\mathrm{CO}_{2}$ incubator at $37^{\circ} \mathrm{C}$

3. Monitor the seeded plates for clonal growth by light microscopy (LM) and change the medium every 3 days.

4. After $10-14$ days of cell growth, wash the Petri dishes twice with PBS and fix the cells in $4 \%$ paraformaldehyde for 30 min at room temperature.

NOTE: Paraformaldehyde is toxic. Please practice caution by wearing gloves and eyewear during use. 
5. Stain the fixed cells with $0.1 \%$ crystal violet for $1 \mathrm{~h}$ at room temperature and rinse the plate with tap water until the unbound blue stain is gone.

6. Count the number of colonies comprised of at least 50 cells using LM.

\section{Expression of cell surface markers}

1. Culture the isolated cells to $70 \%$ confluence, dissociate with the commercial trypsin solution, and wash and pellet by centrifugation at $1,500 \times \mathrm{g}$ for $3 \mathrm{~min}$. Suspend the cells in FACS buffer (1x PBS with $2 \%$ FBS and $0.1 \%$ sodium azide).

NOTE: Sodium azide is toxic. Please practice caution by wearing gloves and eyewear during use.

2. Stain the cells $\left(\sim 10^{6}\right)$ with FITC- or APC-labeled MSC-specific antibodies (FITC-conjugated antibodies against: CD44 and CD90 or APC-conjugated antibodies against: CD29, CD73, and CD105) in FACS buffer for 30 min at $4{ }^{\circ} \mathrm{C}$ in dark.

3. Centrifuge the stained cells at $670 \mathrm{xg}$ for $5 \mathrm{~min}$, aspirate the supernatant, and resuspend the cells in $500 \mu \mathrm{L}$ of FACS buffer.

4. Analyze the antibody-stained cells using FACS according to the manufacturer's protocol.

\section{Differentiation Potential}

\section{Adipogenic differentiation}

1. Culture the isolated cells to $70 \%$ confluence, dissociate with the commercial trypsin solution, and wash and pellet by centrifugation at $1,500 \times \mathrm{g}$ for $3 \mathrm{~min}$.

2. In a $\mathrm{CO}_{2}$ incubator at $37^{\circ} \mathrm{C}$, culture the cells at a concentration of 100,000 cells/well of a 6 -well plate containing $2 \mathrm{~mL}$ of $\mathrm{CM}$.

3. After $24 \mathrm{~h}$, replace the $\mathrm{CM}$ with adipogenic differentiation medium $(0.5 \mu \mathrm{M}$ isobutyl-methylxanthine, $1 \mu \mathrm{M}$ dexamethasone, $10 \mu \mathrm{M}$ insulin, and $200 \mu \mathrm{M}$ indomethacin) and incubate. Change the differentiation medium every 3 days or as often as needed.

4. After 3 weeks of incubation, fix the cells with $2 \mathrm{~mL}$ of $4 \%$ paraformaldehyde per well for 30 min at room temperature and stain with Oil Red $\mathrm{O}$ to detect the production of lipid droplets in order to analyze for adipogenic differentiation.

NOTE: Paraformaldehyde is toxic. Please practice caution by wearing gloves and eyewear during use.

5. Treat the fixed cells with $60 \%$ isopropanol $(2 \mathrm{~mL} /$ well of 6 -well plate) for $5 \mathrm{~min}$.

6. Replace the isopropanol with $2 \mathrm{~mL}$ of Oil Red O stain (filter 3 parts Oil Red O stain and 2 parts distilled water), incubate for 15-30 min at room temperature, and wash 3 - 4 times with tap water to remove any unbound stain.

7. Visualize the red-stained lipid droplets, indicative of adipogenic cell lineage, using LM.

\section{Chondrogenic differentiation}

1. Culture isolated cells to $70 \%$ confluence, dissociate with the commercial trypsin solution, and wash with PBS

2. To pellet cells for chondrogenic induction, centrifuge 250,000 cells in a 15 - $\mathrm{mL}$ centrifuge tube with $2 \mathrm{~mL}$ of $\mathrm{CM}$ at $11,000 \times \mathrm{g}$ for $8 \mathrm{~min}$. Incubate the pellets overnight at $37^{\circ} \mathrm{C}$.

3. After $24 \mathrm{~h}$, gently replace the CM with chondrogenic differentiation medium (20 ng TGF $\beta 1,10 \mathrm{ng}$ insulin, $100 \mathrm{nM}$ dexamethasone, and $100 \mu \mathrm{M}$ ascorbic acid) without disturbing the pellet and continue the incubation. Change the differentiation medium every 3 days or as often as needed.

4. After 3 weeks of incubation, fix the cells with $2 \mathrm{~mL}$ of $4 \%$ paraformaldehyde per well for $30 \mathrm{~min}$ at room temperature. Cryosection and stain with $1 \%$ toluidine blue to examine the presence or production of extracellular matrix in order to determine chondrogenic differentiation.

NOTE: Paraformaldehyde is toxic. Please practice caution by wearing gloves and eyewear during use.

5. For cryosectioning, gently wash the harvested cell pellets twice with PBS and fix with $1 \mathrm{~mL}$ of $4 \%$ paraformaldehyde for 30 min at room temperature. NOTE: Paraformaldehyde is toxic. Please practice caution by wearing gloves and eye wear during use.

6. Wash the pellets twice with PBS to remove the paraformaldehyde and transfer into a sucrose/OCT solution (1:2, 20\% Sucrose:OCT). Incubate at room temperature for $24 \mathrm{~h}$ to allow the solution to completely seep into the cell pellets; this will allow smooth sectioning.

7. Transfer the pellets into the OCT molds. Freeze the molds at $-20^{\circ} \mathrm{C}$ for $4-6 \mathrm{~h}$ and cryosection about $10-12$ micron-thick sections of the cell pellets using a cryostat for toluidine blue staining.

8. Wash the cryosections gently with PBS; add few drops of $1 \%$ toluidine blue stain, covering the pellet sections on the slide completely; and incubate for $1 \mathrm{~h}$ at room temperature.

9. Wash the slides with PBS multiple times to destain. Remove the excess stain by dipping the slide in $\mathrm{HCl}-$ based alcohol solution $(95 \%$ alcohol $+0.5 \mathrm{~mL}$ of $\mathrm{HCl}$ ) multiple times until the unbound blue color is gone. Mount the stained sections using $100 \mu \mathrm{L}$ of mounting solution for the long-term preservation of the slides.

NOTE: The blue staining of the sections indicates the presence of glycosaminoglycans and glycoproteins and will be observable using LM.

\section{Osteogenic differentiation}

1. Again, culture the isolated cells to $70 \%$ confluence, dissociate with the commercial trypsin solution, and wash and pellet by centrifugation at $1,500 \mathrm{xg}$ for $3 \mathrm{~min}$.

2. Subculture the cells at a concentration of 100,000 cells/well of 6 -well plates containing $2 \mathrm{~mL}$ of $\mathrm{CM}$ in a $\mathrm{CO}_{2}$ incubator at $37{ }^{\circ} \mathrm{C}$.

3. After $24 \mathrm{~h}$, replace the $\mathrm{CM}$ with osteogenic differentiation medium $(0.1 \mu \mathrm{M}$ dexamethasone, $10 \mu \mathrm{M} \beta$-glycerophosphate, and $50 \mu \mathrm{M}$ ascorbate phosphate) and continue the incubation. Change the differentiation medium every 3 days or as often as needed.

4. After 3 weeks of incubation, fix the cells with $2 \mathrm{~mL}$ of $4 \%$ paraformaldehyde per well for 30 min at room temperature and stain with alizarin red to detect the presence of a calcium deposition in order to determine osteogenic differentiation. NOTE: Paraformaldehyde is toxic. Please practice caution by wearing gloves and eyewear during use.

5. Gently wash the fixed cells twice with distilled water and treat with $2 \%$ alizarin red stain $(2 \mathrm{~mL} / \mathrm{well}$ in a 6 -well plate $)$ for $1 \mathrm{~h}$ at room temperature in the dark.

6. Remove the excess stain by gently washing with tap water so that the calcium deposits do not dislodge. 
7. Visualize the red-stained calcium deposits using LM.

\section{Quantitative Reverse Transcriptase Polymerase Chain Reaction (qRT-PCR) Analysis}

1. Culture the isolated cells to $70 \%$ confluence, dissociate with the commercial trypsin solution, wash, and pellet by centrifugation at $1,500 \times \mathrm{g}$ for $3 \mathrm{~min}$ to isolate the total cellular RNA.

2. Wash the cells with PBS to remove traces of FBS, and then proceed to the RNA isolation using a commercial RNA purification kit, following the manufacturer's instructions.

3. Purify the isolated total RNA by treating with DNase at $37^{\circ} \mathrm{C}$ for 30 min using a thermocycler. Synthesize cDNA using commercial kit following the manufacturer's instructions.

4. Prepare triplicate $10-\mu \mathrm{L}$ PCR reactions in a 96-well plate, with each reaction containing $5 \mu \mathrm{L}$ of SYBR-green PCR supermix; $3 \mu \mathrm{L}$ of doubledistilled water; $0.5 \mu \mathrm{L}$ of each primer, forward and reverse; and $1 \mu \mathrm{L}$ of the diluted (1:10) cDNA.

5. Perform PCR under the following parameters: $10 \mathrm{~min}$ at $98^{\circ} \mathrm{C}$, followed by 44 cycles of $30 \mathrm{~s}$ each at $98^{\circ} \mathrm{C}, 20 \mathrm{~s}$ at $60{ }^{\circ} \mathrm{C}$, and $30 \mathrm{~s}$ at $72{ }^{\circ} \mathrm{C}$.

6. Normalize the amplification of the target genes to the reference genes, GAPDH and $\beta$-ACTIN; the primer sequences are listed in Table 1.

\section{Statistical Analysis}

1. Perform all analyses using statistical software. Present the data as the mean \pm SEM. Results with a $p$-value $\left({ }^{* *} p \leq 0.01\right.$ and $\left.{ }^{*} p \leq 0.05\right)$ were considered statistically significant.

\section{Representative Results}

Dissection and Isolation of Cells from Human Umbilical Cord, Cord-placenta Junction, and Fetal Placenta

Perinatal tissue consists of three discrete anatomical regions. The first is the UC, containing two arteries and one vein, as well as two distinct zones: CL (the outer lining of the cord) and WJ (the jelly-like material surrounding the blood vessels inside the cord). The second is CPJ (the connection between the cord and the placenta), and the third is FP, which is immediately after the CPJ (the cord inserts into the placenta, which is attached to the uterine wall of the mother). The schematic of the isolation protocol of cells from perinatal tissue is depicted in Figure 1. The four sources-CL, WJ, CPJ, and FP-are clearly identifiable and were separated to isolate cells from the explants, as shown in Figure 2. The appearance of cell outgrowth from explant cultures was routinely monitored and recorded by LM. Adherent fibroblastoid cells were observed after 3-4 days of culturing the CPJ explants. Cells with similar morphology appeared 7-8, 9-10, and 11-14 days after culturing the WJ, CL, and FP explants, respectively. The outgrowth of cells from the explants representing the various sources (CL, WJ, CPJ, and FP) is depicted in Figure 3A. These cells were regarded as P0. When P0 cells were subcultured, they appeared to grow clonally, displaying a fibroblastoid morphology similar to that of BM-MSCs. However, they exhibited variation in the population doubling time, ranging from $32 \mathrm{~h}$ to $94 \mathrm{~h}$ for cells from CPJ and FP, as shown in Figure 3B and C. Cells from WJ and CL had similar doubling times medial to CPJ and FP. Further analysis of the P0 cells indicated that they also varied in the CFE, ranging from 16 to 92 colonies. Cells from the CPJ had the highest (92), while FP cells had the lowest (16) CFE. Cells from CL and WJ had CFE values of 59 and 80 colonies, respectively (Figure 4A-C). The cells from CL had CFE values similar to the BM-MSCs. These results suggest that CPJ-derived cells have higher proliferative and self-renewal capabilities.

\section{Immunoprofile of the Isolated Cells}

Cells isolated from the CL, WJ, CPJ, and FP were investigated for the expression of cell-specific markers. P3-5 cells displayed positive expression for MSCs markers such as CD29, CD44, CD73, CD90, and CD105 (Figure 5A and B). These cells are also known to express major histocompatibility class I marker, HLA-ABC, and do not express class II marker, HLA-DR ${ }^{3}$. The percentages of positive cells for these selected markers from all four sources were similar to that expressed by standard BM-MSCs. However, the MFI ratios indicate that WJ- and CPJ-derived cells are almost similar and are even higher than the CL- and FP-derived cells (Figure 5C). Interestingly, in spite of varying CFE values, all cells from different sources expressed similar levels of MSC markers. Based on the results of cell surface markers, the isolated cells from all four sources were regarded as MSCs. Further analysis of these cells revealed that they also express pluripotency genes, OCT4, NANOG, KLF4, and SOX2, as shown in Figure 5D. The CPJ-MSCs had the highest expression of pluripotency markers, followed by WJ-, CL- and FP-MSCs.

\section{Differentiation Potential of Isolated MSCs}

The gold standard for characterizing MSCs is their ability to differentiate into multiple lineages. Our results showed that isolated MSCs from all of the perinatal sources readily differentiated into adipogenic, chondrogenic, and osteogenic cell types. Adipogenic derivatives produced lipid droplets that were positively stained with Oil Red O, as shown in Figure 6A. Toluidine blue staining of the chondrogenic derivative of MSCs demonstrated the presence of proteoglycans and glycoproteins that aided in the production of extracellular matrix (Figure 6B). Osteogenic derivatives of MSCs positively stained with alizarin red indicate the presence of the calcium deposits involved in bone mineralization, as shown in Figure 6C. 
As expected, the transcriptional analysis of isolated MSCs from all sources showed trilineage differentiation (Figure 6D-F). However, the potential of differentiation varied depending on the source of MSCs. Adipogenic derivatives of WJ-MSCs had 2-fold greater expression levels of the selected adipogenic genes (CEBP $\beta$, FABP4, and PPARY) compared to CPJ-MSCs. CL- and FP-MSCs had the lowest expression of these genes. In the case of chondrogenic differentiation, CPJ-MSCs derivatives expressed selected chondrogenic genes (SOX9 and COL2) 50-fold higher than the WJ- and FP-MSCs derivatives. Similar to the poor adipogenic differentiation of CL-MSCs, they had lower chondrogenic potential, as evident by the lowest expression of chondrogenic genes. CPJ-MSCs also showed the highest osteogenic differentiation potential, as indicated by the 2-fold higher transcript levels of selected osteogenic genes (COL1, OPN, and OCN). However, the expression of progenitor marker RUNX2 was highest in osteogenic derivatives of WJ-MSCs, indicating that these cells were slow in responding to differentiation conditions. Again, CL-MSCs showed poor differentiation into osteogenic lineage. These results suggest that CPJ-MSCs and WJ-MSCs displayed greater differentiation potential than CL- and FP-MSCs. The CL-MSCs had the lowest potential to differentiate into trilineage derivatives.

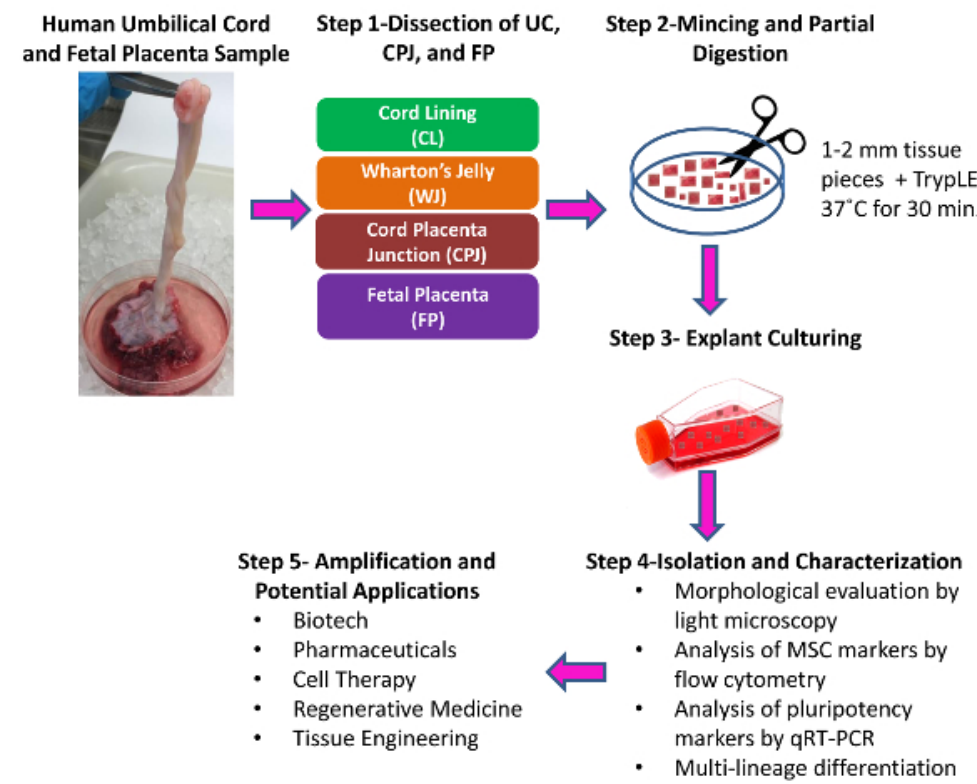

Figure 1: Schematic of the Isolation of Cells from Human Umbilical Cord, Cord-placenta Junction, and Fetal Placenta. Inspect the collected sample and proceed with the dissection. Step 1. Manually dissect and separate the cord/placenta sample into three discrete regions: umbilical cord (UC), cord-placenta junction (CPJ), and fetal placenta (FP). Separate the two distinct zones of the UC into cord lining (CL) and Wharton's Jelly (WJ) using scissors and forceps. Step 2. Cut each of the dissected tissues separately into 1- to 2-mm pieces using scissors and partially digest the pieces. Step 3. Culture the tissue pieces. Step 4. Isolate the cells from the explants by trypsin treatment. Subculture and characterize the isolated cells. Step 5. Isolated and characterized cells can be amplified and used for various applications. Please click here to view a larger version of this figure. 


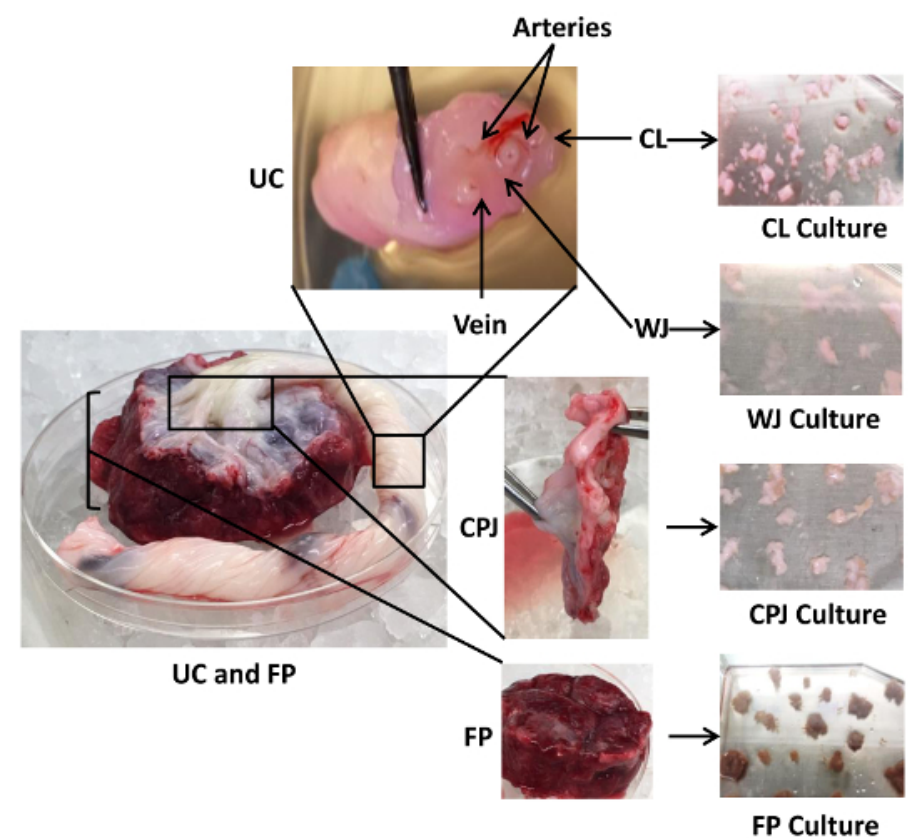

Figure 2: Dissection and Culture of Explants from Human Umbilical Cord, Cord-placenta Junction, and Fetal Placenta. Shown are three different anatomical regions of the cord/placenta sample: UC (split into the CL and WJ), CPJ, and FP, as well as the associated arteries and veins. CL, WJ, CPJ, and FP were separated by manual dissection to isolate cells from the explants. Each of the dissected regions was separately cut into small fragments and cultured in $75-\mathrm{cm}^{2}$ plates using $9 \mathrm{~mL}$ of $\mathrm{CM}$. Please click here to view a larger version of this figure.

A

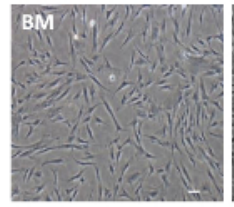

C

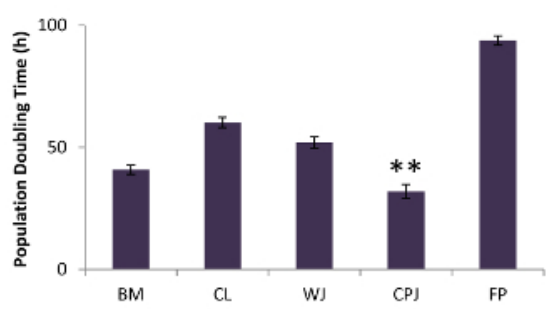

Figure 3: Morphology of Isolated Cells from Human Umbilical Cord, Cord-placenta Junction, and Fetal Placenta. (A) Cell outgrowth from the explants of CL, WJ, CPJ, and FP, as visualized by LM. (B) Subculture of the isolated cells from CL, WJ, CPJ, and FP explants showed fibroblastoid morphology. The scale bar represents $100 \mu \mathrm{m}$ (magnification: 4X). (C) Population doubling time of the isolated cells from CL, WJ, $\mathrm{CPJ}$, and FP. BM-MSCs were used as a standard. The error bars represent the standard error of the mean of the triplicate measurements ${ }^{* *} \mathrm{p} \leq$ 0.01 and $\left.{ }^{*} p \leq 0.05\right)$. Please click here to view a larger version of this figure. 
BM

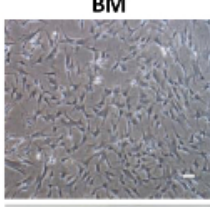

B
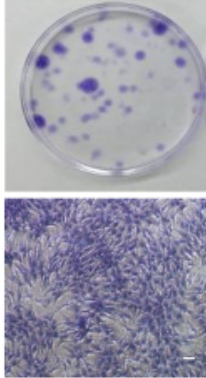

$\mathrm{CL}$
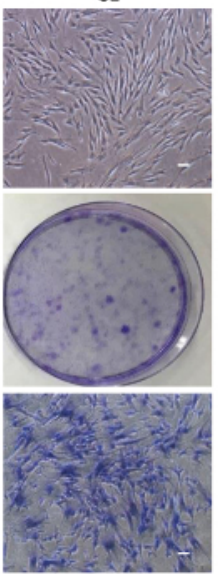

D

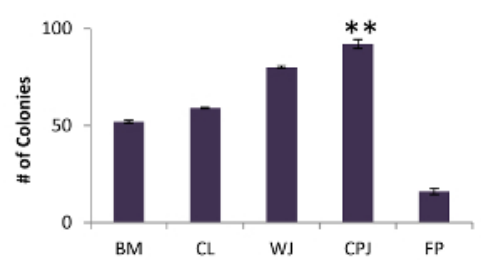

WJ
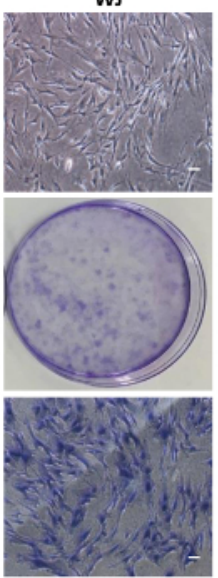

CPJ

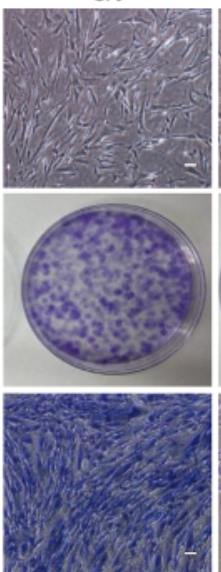

FP

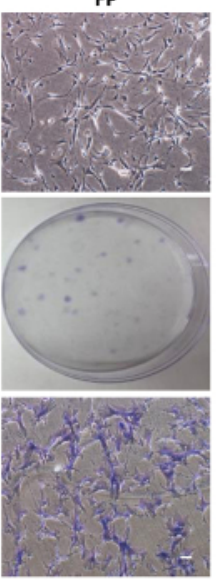

Figure 4: Colony-forming Efficiency of Cells from Human Umbilical Cord, Cord-placenta Junction, and Fetal Placenta. (A) Growth of the cells (P0) isolated from CL, WJ, CPJ, and FP, plated at a clonal density of $1.63 \mathrm{cells} / \mathrm{cm}^{2}$, were visualized by LM. (B) Photomicrographs of cells stained with crystal violet displaying colony-forming capacity. (C) Single colony of cells stained with crystal violet. The scale bars represent $100 \mu \mathrm{m}$ (magnification: 4X). (D) Graphical representation of the number of colonies formed from the cells derived from CL, WJ, CPJ, and FP. BM-MSCs were used as a standard. The error bars represent the standard error of the mean of the triplicate measurements $\left({ }^{\star *} p \leq 0.01\right.$ and ${ }^{*} p \leq$ 0.05). Please click here to view a larger version of this figure. 

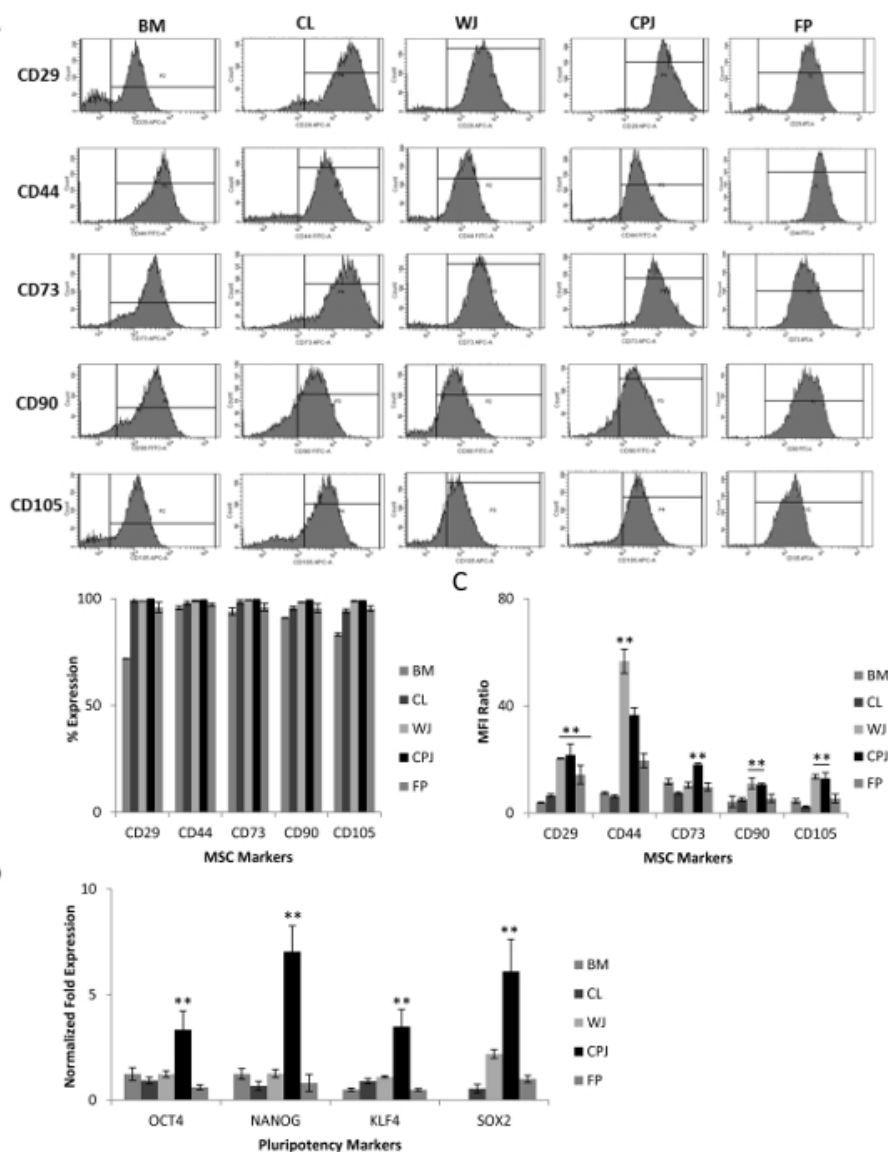

Figure 5: Analysis of MSC Markers Expressed by the Cells Isolated from Human Umbilical Cord, Cord-placenta Junction, and Fetal Placenta. (A) Expression of mesenchymal surface markers (CD29, CD44, CD73, CD90, and CD105) by the cells from CL, WJ, CPJ, and FP, as determined by flow cytometry. (B and C) Graphical representation of the percentages of positive cells and the median fluorescent intensity (MFI) ratios for the selected mesenchymal surface markers. MFI ratios were calculated by dividing the MFI value of the marker (generated by the software based on the fluorescence intensity of gated cell populations) by the MFI value of the isotype. The error bars represent the standard error of the mean of the triplicate measurements. (D) Expression of the pluripotency genes (OCT4, NANOG, KLF4, and SOX2) by the cells from $\mathrm{CL}$, WJ, CPJ, and FP, as determined by qRT-PCR. ( ${ }^{* *} \mathrm{p} \leq 0.01$ and $\left.{ }^{*} \mathrm{p} \leq 0.05\right)$. Gene expression was normalized to GAPDH and $\beta-A C T I N$, and the error bars represent the standard error of the mean of the triplicate measurements. Please click here to view a larger version of this figure. 
A

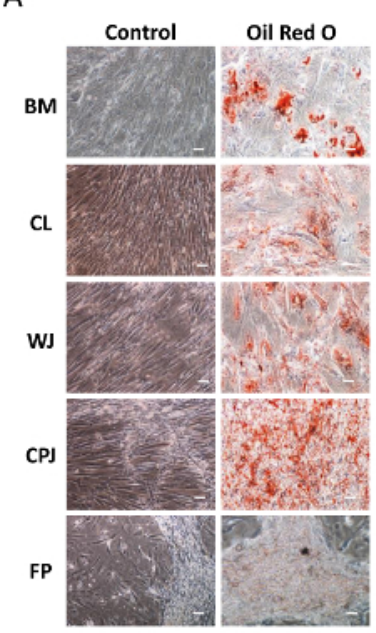

D

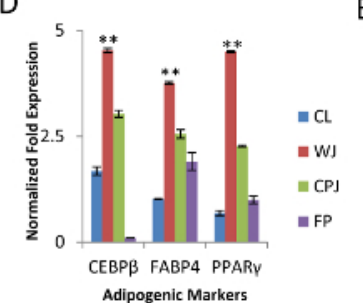

B

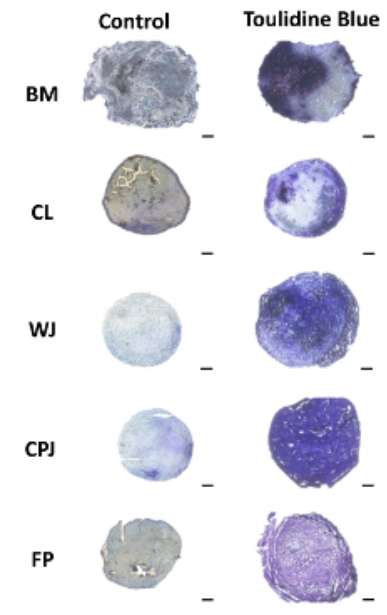

E

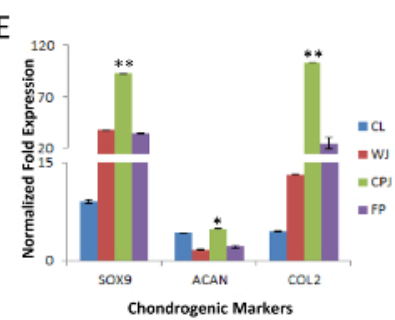

C

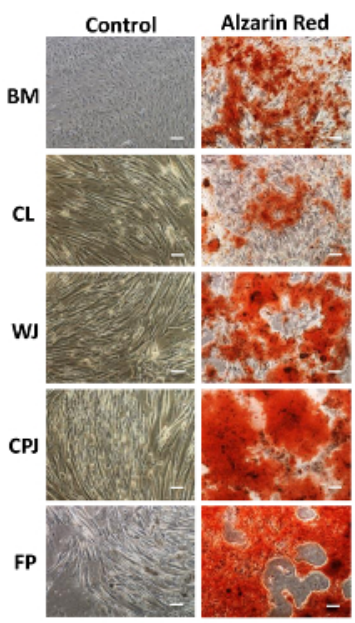

$\mathrm{F}$

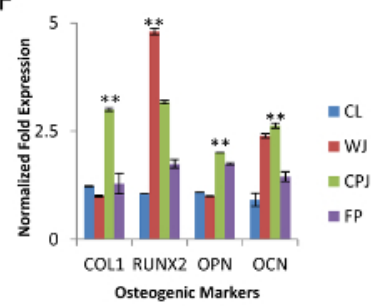

Figure 6: Multilineage Differentiation of MSCs Isolated from Human Umbilical Cord, Cord-placenta Junction, and Fetal Placenta. (A) Cells were incubated in adipogenic differentiation medium for 3 weeks and stained with Oil Red O, indicating the presence of lipid droplets. (B) Cell pellets were incubated in chondrogenic differentiation medium for 3 weeks. Histological cryosections of pellet cultures were stained with toluidine blue, displaying the presence of glycosaminoglycans. (C) Cells were incubated in osteogenic differentiation medium for 3 weeks and stained with alizarin red, displaying the production of calcium deposits suggesting bone mineralization. All images were obtained by LM. The scale bar represents $100 \mu \mathrm{m}$ (magnification: 4X). BM-MSCs were used as a standard. (D-F) Expression of selected genes (CEBP $\beta$, FABP4, and PPARY; SOX9, ACAN, and COL2; and COL1, RUNX2, OPN, and OCN representing the differentiation of MSCs into adipogenic, chondrogenic, and osteogenic lineages, respectively), as determined by qRT-PCR analysis ( ${ }^{* *} p \leq 0.01$ and $\left.{ }^{*} p \leq 0.05\right)$. Gene expression was normalized to GAPDH and $\beta$-ACTIN, and error bars represent the standard error of the mean of the triplicate measurements. Please click here to view a larger version of this figure. 


\begin{tabular}{|c|c|c|}
\hline Gene & Primer Sequences & Product Length \\
\hline \multirow[t]{2}{*}{ OCT4 } & Forward-CCCCTGGTGCCGTGAA & 97 \\
\hline & Reverse-GCAAATTGCTCGAGTTCTTTCTG & \\
\hline \multirow[t]{2}{*}{ NANOG } & Forward- AAAGAATCTTCACCTATGCC & 110 \\
\hline & Reverse- GAAGGAAGAGGAGAGACAGT & \\
\hline \multirow[t]{2}{*}{ KLF4 } & Forward- CGAACCCACACAGGTGAGAA & 94 \\
\hline & Reverse- TACGGTAGTGCCTGGTCAGTTC & \\
\hline \multirow[t]{2}{*}{ sox2 } & Forward- TTGCTGCCTCTTTAAGACTAGGA & 75 \\
\hline & Reverse- CTGGGGCTCAAACTTCTCTC & \\
\hline \multirow[t]{2}{*}{ sox9 } & Forward- AGCGAACGCACATCAAGAC & 85 \\
\hline & Reverse- CTGTAGGCGATCTGTTGGGG & \\
\hline \multirow[t]{2}{*}{ COL2 } & Forward- CTCGTGGCAGAGATGGAGAA & 252 \\
\hline & Reverse- CACCAGGTTCACCAGGATTG & \\
\hline \multirow[t]{2}{*}{ ACAN } & Forward- AGCCTGCGCTCCAATGACT & 103 \\
\hline & Reverse- GGAACACGATGCCTTTCACC & \\
\hline \multirow[t]{2}{*}{ COL1 } & Forward- AAGGTCATGCTGGTCTTGCT & 114 \\
\hline & Reverse- GACCCTGTTCACCTTTTCCA & \\
\hline \multirow[t]{2}{*}{ RUNX2 } & Forward- TGCTTCATTCGCCTCACAAA & 111 \\
\hline & Reverse- AGTGACCTGCGGAGATTAAC & \\
\hline \multirow[t]{2}{*}{ OPN } & Forward- CATACAAGGCCATCCCCGTT & 112 \\
\hline & Reverse- TGGGTTTCAGCACTCTGGTC & \\
\hline \multirow[t]{2}{*}{$\mathrm{OCN}$} & Forward- TAAACAGTGCTGGAGGCTGG & 191 \\
\hline & Reverse- CTTGGACACAAAGGCTGCAC & \\
\hline \multirow[t]{2}{*}{ CEBP $\beta$} & Forward- TATAGGCTGGGCTTCCCCTT & 94 \\
\hline & Reverse- AGCTTTCTGGTGTGACTCGG & \\
\hline \multirow[t]{2}{*}{ FABP4 } & Forward- TTAGATGGGGGTGTCCTGGT & 158 \\
\hline & Reverse- GGTCAACGTCCCTTGGCTTA & \\
\hline \multirow[t]{2}{*}{ PPARY } & Forward- GGCTTCATGACAAGGGAGTTTC & 74 \\
\hline & Reverse- ACTCAAACTTGGGCTCCATAAAG & \\
\hline \multirow[t]{2}{*}{ GAPDH } & Forward- ACAACTTTGGTATCGTGGAAGG & 101 \\
\hline & Reverse- GCCATCACGCCACAGTTTC & \\
\hline \multirow[t]{2}{*}{ ACTIN } & Forward- AATCTGGCACCACACCTTCTAC & 170 \\
\hline & Reverse- ATAGCACAGCCTGGATAGCAAC & \\
\hline
\end{tabular}

Table 1: List of Human Primer Sequences Used in this Study.

\section{Discussion}

Recent advances in stem cell research not only improved the understanding of basic development processes but also provided promising opportunities for the use of stem cells in biotechnological, pharmaceutical, cell therapy, regenerative medicine, and tissue engineering applications $s^{18,19,20}$. While pluripotent ESCs isolated from early embryos are the most promising, they face technical challenges and ethical dilemmas $^{21,22,23}$. Induced pluripotent stem cells (iPSCs) derived from adult cells provide an alternative, but they too face similar technical and safety problems ${ }^{23}$. Furthermore, iPSCs may not be genetically stable or may have undergone genetic changes, thus limiting their therapeutic use. Stem cells have also been reported in a variety of postnatal tissues and organs. The most common sources of these ASCs are the bone marrow, adipose, and muscle tissue. However, ASCs from postnatal sources have limited growth and differentiation potential ${ }^{24,25}$. They also suffer due to aging and exposure to environmental stresses and thus are not always efficacious for therapeutic applications ${ }^{26,27,28}$. This has led us and others to search for new sources of stem cells that are more naïve than ASCs.

We have investigated perinatal sources for primitive stem cells as an alternative to ASCs. In this report, we provide a reliable, robust, and simple method to isolate human MSCs from cord/placenta samples. In comparison to other sources and methods used for the isolation of ASCs, this 
method provides an efficient and non-invasive method for isolating large quantities of high-quality MSCs. It further accentuates the higher growth and differentiation potential of perinatal MSCs compared to MSCs from adult sources such as BM.

The UC attaching the fetus to the placenta is a large organ with distinct anatomical regions, including two arteries, a vein, CL, and WJ (tissue surrounding the blood vessels). Several studies have reported the isolation of MSCs from the whole cord, the WJ, or the placenta, with variable growth and differentiation potentials ${ }^{25,29}$. Nevertheless, to date, no study has effectively investigated and compared the cells from different anatomical regions of the cord/placenta sample. We provide here a systematic and simple method for the dissection of the UC, CPJ, and connected FP for the isolation of MSCs. We also show a comprehensive and simple protocol to characterize and evaluate the quality of the isolated cells by determining their proliferation capabilities, as well as their self-renewal and differentiation potentials. This method is reproducible and yields a large amount of superior-quality MSCs.

We found that the partial digestion of tissue pieces 1-2 mm in size using a commercial trypsin solution reproducibly yielded cells from the explants, whereas the complete digestion of tissue into single cells had poor yields of isolated cells. However, one study reported that larger tissue explants of UC approximately $10 \mathrm{~mm}$ in size were optimal for cell isolation ${ }^{30}$. Conversely, another study suggested that the tissue explant method resulted in a longer culture cycle and lower yield of cells compared to the enzyme digestion method ${ }^{31}$. In previous reports, the treatment of cord tissues with collagenase II and hyaluronidase, separately or following the trypsin, have been performed to isolate cord cells ${ }^{32,33,34}$. Not only is there a great deal of variation in digestion methods of the cord tissue prior to culturing, but also the isolated cells appeared to be heterogeneous. The use of harsh enzymes for longer time periods may degrade the cell membrane, affecting cell adherence and proliferation. The results of this method showed that partially-digested perinatal tissue explants provided outgrowth of cells without causing extensive cell damage, maintained viability, and yielded higher amounts of homogeneous populations of MSCs.

While the protocol for the dissection and isolation of cells from the explants is straightforward, some of the steps may prove to be challenging. First, ensure explant adherence by allowing their attachment to the surface of the culture flask. This can be facilitated by using a small amount of medium, only covering the tissue pieces for the first $2 \mathrm{~h}$ of culture, and then carefully adding the remaining medium. Second, limit the number of explants to less than 15 per T75 flask. Too little space between the pieces or too many pieces per flask are inhibitory for cell outgrowth. Third, tissue pieces that do not stick to the surface of the culture flask after 3 days of incubation should be transferred to a new flask for adherence and culturing. Fourth, tissue pieces to be cultured should be free of cell debris, which inhibits attachment. Fifth, the culturing of large pieces requires more medium and does not improve cell outgrowth efficiency. Lastly, monitor the explant cultures closely, particularly after the appearance of cell outgrowth, as the cells may rapidly become confluent and differentiate depending upon the tissue source. A few limitations of the technique include a lack of expertise in dissecting the samples to separate the CL, WJ, CPJ, and FP; a small sample size, resulting in low WJ yield; and delayed processing-the samples are required to be processed within 2-4 $\mathrm{h}$ of collection to avoid a loss in cell recovery.

The gold standard for the characterization of MSCs is the ability to adhere to plastic, form the fibroblastic phenotype, and differentiate into multiple lineages. These are also commonly-used criteria for defining MSCs as described by the ISCT ${ }^{35}$. In this study, cells isolated from all four sources were adherent to plastic and had positive expression for MSC markers (CD29, CD44, CD73, CD90, and CD105) but negative expression for the hematopoietic marker CD45. In addition, they expressed human leukocyte antigen (HLA) class I but were negative for HLA class II. Compared to the BM-MSCs, WJ- and CPJ-derived cells were higher. These results are consistent with the previous reports of UCderived MSCs ${ }^{33,36,37,38}$. In addition, our results showed that CL-, WJ-, CPJ-, FP-MSCs expressed pluripotency genes such as OCT4, NANOG, and SOX2; however, their expression was lower than that of ESCs, as reported previously ${ }^{39}$. These results were also in agreement with a previous study that reported the expression of pluripotency markers in perinatal-derived cells ${ }^{40}$. Interestingly, it was observed that the expression of pluripotent marker was higher in CPJ-MSCs than in cells isolated from other sources. It was also noticed that the UC-MSCs exhibited greater self-renewal potential than BM-MSCs ${ }^{41}$. Interestingly, despite similarity in phenotypic characteristics, the cells isolated from the four sources had variable CFE values. However, except for FP-MSC, they all had better CFE values than BM-MSCs. CPJ-MSCs had the highest CFE value and rate of proliferation when compared to all other MSCs. In addition, WJ- and CPJ-MSCs displayed higher differentiation potentials than BM-MSCs. CL-MSCs showed the least differentiation potential into all three lineages (i.e., adipogenic, chondrogenic, and osteogenic), whereas CPJ-MSCs had the highest trilineage differentiation potential and FP-MSCs displayed the least adipogenic differentiation potential.

In conclusion, our results showed that the quality and quantity of isolated MSCs differed between the four sources in the cord/placenta sample. CPJ-MSCs had more proliferation capacity and greater self-renewal potential. They were also potent in their differentiation into the trilineage cell types. Due to their low doubling time, CPJ-MSCs could be rapidly expanded 1,000-fold and used for high-throughput drug or biomaterial screening. These cells could be a more promising source for cell therapy and regenerative medicine applications.

\section{Disclosures}

The authors have nothing to disclose.

\section{Acknowledgements}

The study was supported by OU-WB ISCRM, Oakland University and Michigan Head and Spine Institute. N. Beeravolu and C. McKee received the Provost Graduate Research Award from Oakland University. We appreciate S. Bakshi for reviewing the manuscript.

1. Upadhyay, R. K. Role of regeneration in tissue repairing and therapies. J Regen Med Tissue Eng. 4 (1), 1 (2015).

2. English, K., Mahon, B. P., \& Wood, K. J. Mesenchymal stromal cells; role in tissue repair, drug discovery and immune modulation. Curr Drug Deliv. 11 (5), 561-571 (2014).

3. Pak, J., Lee, J. H., \& Lee, S. H. Regenerative repair of damaged meniscus with autologous adipose tissue-derived stem cells. Biomed Res Int. 2014436029 (2014). 
4. Hocking, A. M. Mesenchymal Stem Cell Therapy for Cutaneous Wounds. Adv Wound Care (New Rochelle). 1 (4), $166-171$ (2012).

5. Anthony, D. F., \& Shiels, P. G. Exploiting paracrine mechanisms of tissue regeneration to repair damaged organs. Transplant Res. 2 (1), 10 (2013).

6. Teschendorff, A. E. et al. Epigenetic variability in cells of normal cytology is associated with the risk of future morphological transformation. Genome Med. 4 (3), 24 (2012).

7. Liu, L. et al. Chromatin modifications as determinants of muscle stem cell quiescence and chronological aging. Cell Rep. 4 (1), 189-204 (2013).

8. Bentivegna, A. et al. DNA Methylation Changes during In Vitro Propagation of Human Mesenchymal Stem Cells: Implications for Their Genomic Stability? Stem Cells Int. 2013192425 (2013).

9. Bieback, K., \& Brinkmann, I. Mesenchymal stromal cells from human perinatal tissues: From biology to cell therapy. World J Stem Cells. 2 (4), 81-92 (2010).

10. Tobita, M., Tajima, S., \& Mizuno, H. Adipose tissue-derived mesenchymal stem cells and platelet-rich plasma: stem cell transplantation methods that enhance stemness. Stem Cell Res Ther. 6215 (2015).

11. Kim, D. W. et al. Wharton's jelly-derived mesenchymal stem cells: phenotypic characterization and optimizing their therapeutic potential for clinical applications. Int J Mol Sci. 14 (6), 11692-11712 (2013).

12. Baraniak, P. R., \& McDevitt, T. C. Stem cell paracrine actions and tissue regeneration. Regen Med. 5 (1), 121-143 (2010).

13. Prado, S. et al. Human amniotic membrane as an alternative source of stem cells. Differentiation. 1 162-171 (2011).

14. Puissant, B. et al. Immunomodulatory effect of human adipose tissue-derived adult stem cells: comparison with bone marrow mesenchymal stem cells. Br J Haematol. 129 (1), 118-129 (2005).

15. Christodoulou, I., Kolisis, F. N., Papaevangeliou, D., \& Zoumpourlis, V. Comparative Evaluation of Human Mesenchymal Stem Cells of Fetal (Wharton's Jelly) and Adult (Adipose Tissue) Origin during Prolonged In Vitro Expansion: Considerations for Cytotherapy. Stem Cells Int. 2013246134 (2013).

16. Dalous, J., Larghero, J., \& Baud, O. Transplantation of umbilical cord-derived mesenchymal stem cells as a novel strategy to protect the central nervous system: technical aspects, preclinical studies, and clinical perspectives. Pediatr Res. 71 (4 Pt 2), $482-490$ (2012).

17. Escacena, N., Quesada-Hernandez, E., Capilla-Gonzalez, V., Soria, B., \& Hmadcha, A. Bottlenecks in the Efficient Use of Advanced Therapy Medicinal Products Based on Mesenchymal Stromal Cells. Stem Cells Int. 2015 (2015).

18. Law, S., \& Chaudhuri, S. Mesenchymal stem cell and regenerative medicine: regeneration versus immunomodulatory challenges. Am J Stem Cells. 2 (1), 22-38 (2013).

19. Brower, J. et al. Mesenchymal stem cell therapy and delivery systems in nonhealing wounds. Adv Skin Wound Care. 24 (11), 524-532 (2011).

20. Angelos, M. G., \& Kaufman, D. S. Pluripotent stem cell applications for regenerative medicine. Curr Opin Organ Transplant. 20 (6), $663-670$ (2015).

21. Pera, M. F. Scientific considerations relating to the ethics of the use of human embryonic stem cells in research and medicine. Reprod Fertil Dev. 13 (1), 23-29 (2001).

22. Espinoza, N., \& Peterson, M. How to depolarise the ethical debate over human embryonic stem cell research (and other ethical debates too!). J Med Ethics. 38 (8), 496-500 (2012).

23. Barrilleaux, B., \& Knoepfler, P. S. Inducing iPSCs to escape the dish. Cell Stem Cell. 9 (2), 103-111 (2011).

24. Ding, D. C., Chang, Y. H., Shyu, W. C., \& Lin, S. Z. Human umbilical cord mesenchymal stem cells: a new era for stem cell therapy. Cell Transplant. 24 (3), 339-347 (2015).

25. Marc G. Jeschke, G. G. G., Thang T. Phan, David N. Herndon and Katsuhiro Kita. Umbilical Cord Lining Membrane and Wharton's JellyDerived Mesenchymal Stem Cells: the Similarities and Differences. J Tissue Eng Regen Med. 4 21-27 (2011).

26. Oh, J., Lee, Y. D., \& Wagers, A. J. Stem cell aging: mechanisms, regulators and therapeutic opportunities. Nat Med. 20 (8), $870-880$ (2014).

27. Burkhalter, M. D., Rudolph, K. L., \& Sperka, T. Genome instability of ageing stem cells-Induction and defence mechanisms. Ageing Res Rev. 23 (Pt A), 29-36 (2015).

28. Adams, P. D., Jasper, H., \& Rudolph, K. L. Aging-Induced Stem Cell Mutations as Drivers for Disease and Cancer. Cell Stem Cell. 16 (6), 601-612 (2015).

29. Seshareddy, K., Troyer, D., \& Weiss, M. L. Method to isolate mesenchymal-like cells from Wharton's Jelly of umbilical cord. Methods Cell Biol. 86 101-119 (2008)

30. Trivanovic, D. et al. Mesenchymal stem cells isolated from peripheral blood and umbilical cord Wharton's jelly. Srp Arh Celok Lek. 141 (3-4), 178-186 (2013).

31. Yoon, J. H. et al. Comparison of explant-derived and enzymatic digestion-derived MSCs and the growth factors from Wharton's jelly. Biomed Res Int. 2013428726 (2013).

32. Steigman, S. A., \& Fauza, D. O. Isolation of mesenchymal stem cells from amniotic fluid and placenta. Curr Protoc Stem Cell Biol. Chapter 1 (2007).

33. Mennan, C. et al. Isolation and characterisation of mesenchymal stem cells from different regions of the human umbilical cord. Biomed Res Int. 2013 (2013).

34. Maria Teresa Conconi, Rosa Di Liddo, Mara Tommasini, Chiara Calore and \& Parnigotto, P. P. Phenotype and differentiation potential of stem populations obtained from various zones of human umbilical cord-an overview. J Tissue Eng Regen Med. 4 6-20 (2011).

35. Dominici, M. et al. Minimal criteria for defining multipotent mesenchymal stromal cells. The International Society for Cellular Therapy position statement. Cytotherapy. 8 (4), 315-317 (2006).

36. Weiss, M. L. et al. Human umbilical cord matrix stem cells: preliminary characterization and effect of transplantation in a rodent model of Parkinson's disease. Stem Cells. 24 (3), 781-792 (2006).

37. Sarugaser, R., Lickorish, D., Baksh, D., Hosseini, M. M., \& Davies, J. E. Human umbilical cord perivascular (HUCPV) cells: a source of mesenchymal progenitors. Stem Cells. 23 (2), 220-229 (2005).

38. Durnaoglu, S., Genc, S., \& Genc, K. Patient-specific pluripotent stem cells in neurological diseases. Stem Cells Int. 2011212487 (2011).

39. Beeravolu, N. et al. Isolation and comparative analysis of potential stem/progenitor cells from different regions of human umbilical cord. Stem Cell Res. 16 (3), 696-711 (2016).

40. Nekanti, U. et al. Long-term expansion and pluripotent marker array analysis of Wharton's jelly-derived mesenchymal stem cells. Stem Cells Dev. 19 (1), 117-130 (2010). 
41. Nagamura-Inoue, T., \& He, H. Umbilical cord-derived mesenchymal stem cells: Their advantages and potential clinical utility. World $J$ Stem Cells. 6 (2), 195-202 (2014). 